\title{
The Meckel-Gruber Syndrome protein TMEM67 (meckelin) regulates basal body planar polarization and non-canonical Wnt signalling via Wnt5a and ROR2
}

\author{
Z Abdelhamed ${ }^{1,2^{*}}$, S Natarajan², C Inglehearn ${ }^{2}$, C Toomes $^{2}$, C Johnson², D Jagger ${ }^{3}$ \\ From Cilia 2014 - Second International Conference \\ Paris, France. 18-21 November 2014
}

\section{Objective:}

Ciliopathies are a group of developmental disorders that manifest with multi-organ anomalies. Mutations in TMEM67 have been reported in human ciliopathies that include Meckel-Gruber and Joubert syndromes. In this study we describe multi-organ developmental abnormalities in the Tmem6 $67^{\text {tm1Dgen/H1 }}$ knockout mouse that closely resemble those of Wnt5a and Ror 2 knockout mice.

\section{Methods:}

We used anatomical assessment, immunofluorescence confocal microscopy and biochemical methods to determine mutant phenotypes at the organismal, cellular and molecular levels.

\section{Results:}

Tmem $67^{\prime-}$ mutant phenotypes include pulmonary hypoplasia, ventricular septal defects, shortening of the body longitudinal axis, limb abnormalities, and cochlear hair cell stereociliary bundle orientation and basal body/ kinocilium positioning defects. The basal body/kinocilium complex was often uncoupled from the hair bundle, suggesting aberrant basal body migration. TMEM67 (meckelin) is essential for phosphorylation of the noncanonical Wnt receptor ROR2 (receptor tyrosine kinaselike orphan receptor 2) upon Wnt5a stimulation. ROR2 interacts with the intracellular C-terminal domain of TMEM67 and co-localizes with TMEM67 at the ciliary transition zone. The N-terminal domain of TMEM67 preferentially binds to Wnt5a in an in vitro binding assay. Tmem67 mutant embryonic lungs in ex vivo culture failed to respond to Wnt5a stimulation of epithelial morphogenesis. However, stimulating the non-canonical Wnt pathway downstream of the receptor by activating RhoA resulted in an elicited response and the rescue of lung hypoplasia phenotypes.

\section{Conclusion:}

Our data suggest that TMEM67 is a novel receptor that has a major role in non-canonical Wnt signalling by Wnt5a and ROR2. We propose that this signalling ensures correct basal body positioning.

\section{Authors' details}

${ }^{1}$ Anatomy and Embryology Department, Al-Azhar University, Cairo, Egypt. ${ }^{2}$ Ophthalmology and Neuroscience, University of Leeds, Leeds, UK. ${ }^{3}$ Ear Institute, University College London, London, UK.

Published: 13 July 2015

\section{doi:10.1186/2046-2530-4-S1-P40}

Cite this article as: Abdelhamed et al:: The Meckel-Gruber Syndrome protein TMEM67 (meckelin) regulates basal body planar polarization and non-canonical Wnt signalling via Wnt5a and ROR2. Cilia 2015 4(Suppl 1):P40. 